\title{
(2) OPEN ACCESS
}

\section{Endoscopic full-thickness plication for the treatment of PPI-dependent GERD: results from a randomised, sham controlled trial}

\author{
Rakesh Kalapala (1) , ${ }^{1}$ Arun Karyampudi, ${ }^{1}$ Zaheer Nabi, ${ }^{1}$ Santosh Darisetty, ${ }^{2}$ \\ Nitin Jagtap $0{ }^{3}{ }^{3}$ Mohan Ramchandani, ${ }^{4}$ Rajesh Gupta, ${ }^{5}$ Sundeep Lakhtakia, \\ Rajesh Goud ${ }_{1}^{6}$ G Venkat Rao, ${ }^{7}$ Prateek Sharma, ${ }^{8}$ D Nageshwar Reddy ${ }^{9}$
}

- Additional supplemental material is published online only. To view, please visit the journal online (http://dx.doi.org/ 10.1136/gutjnl-2020-321811).

For numbered affiliations see end of article.

Correspondence to Dr Rakesh Kalapala, Medical Gastroenterology, Asian Institute of Gastroenterology, Hyderabad 500082, India; drkalpala@gmail.com

Received 13 May 2020 Revised 30 March 2021 Accepted 1 April 2021
Check for updates

(c) Author(s) (or their employer(s)) 2021. Re-use permitted under CC BY-NC. No commercial re-use. See rights and permissions. Published by BMJ.

To cite: Kalapala $\mathrm{R}$

Karyampudi A, Nabi Z, et al. Gut Epub ahead of print: [please include Day Month Year]. doi:10.1136/

gutjnl-2020-321811

\section{ABSTRACT}

Background The majority of endoscopic antireflux procedures for GERD are cumbersome to use and randomised long-term data are sparse. We conducted such a trial to determine the efficacy and safety of a novel, easy to use endoscopic full-thickness fundoplication (EFTP) device in patients with GERD.

Design Patients with proton pump inhibitor (PPI)dependent GERD were randomised to either EFTP or a sham procedure in 1:1 ratio. The primary endpoint was $\geq 50 \%$ improvement in the health-related quality of life (GERD-HRQL) score at 3 months. Secondary end points included improvement in GERD-HRQL, reflux symptom scores, PPI usage, oesophageal acid exposure and reflux episodes and endoscopic findings at 3, 6 and 12 months. Results Seventy patients were randomised; 35 in each group with a median (IQR) age of $36(29-42)$ years, $71.4 \%$ males. $70 \%$ had non-erosive reflux disease on endoscopy with a mean DeMeester score of 18.9 $( \pm 19.93)$. The mean $( \pm S D)$ duration of EFTP procedure was $17.4( \pm 4) \mathrm{min}$. The primary end point was more frequently achieved in the EFTP group (65.7\% vs $2.9 \%$; $\mathrm{p}<0.001$ ). Median (IQR) \% improvement in GERD-HRQL was significantly higher in the EFTP group at 6 (81.4 $(60.9-100.0)$ versus $8.0(2.2-21.6) ; p<0.001)$ and 12 (92.3 (84.4-100.0) versus 9.1 (4.8-36.0); $p<0.001)$ months. In the EFTP group, $62.8 \%$ patients were off-PPI at 12 months compared with $11.4 \%$ in the sham group $(p<0.001)$. pH-metry parameters partially improved at 3 months, $(n=70$; total reflux episodes in EFTP arm and non-acid reflux episodes for EFTP vs sham) but not at 12 months ( $n=27)$; endoscopic oesophagitis was seen in $0 \%$ in the treatment $(n=18)$ and $5(29.4 \%)$ in the control group ( $n=17)$ at 12 months. No major procedure-related adverse events were encountered in either group.

Conclusion EFTP using a novel device is safe and effective in improving quality of life in patients with PPI dependent mostly non-erosive reflux disease at short and long terms; objective parameters showed a limited response rate.

Trial registration number NCT03322553.

\section{INTRODUCTION}

GERD is one among the most common GI problems with a global prevalence of $8 \%-33 \% .^{1}$ The disease-related symptoms such as heartburn,

\section{Significance of this study}

What is already known about this subject?

- Endoscopic fundoplication is a minimally invasive antireflux therapy in patients with proton pump inhibitor (PPI) dependence who refuse surgery; however, the majority of the endoscopic devices are cumbersome to use and robust data on their long-term efficacy are lacking

What are the new findings?

- Our study found endoscopic full-thickness fundoplication, using a novel device, was safe and significantly improved GERD-related quality of life and severity of reflux symptoms at short and long terms, compared with a sham procedure.

How might it impact on clinical practice in the foreseeable future?

- This endoluminal procedure with a short operating time and very few side effects is a promising alternative option to surgery in appropriately selected group of patients, who may not want to continue PPI long-term.

regurgitation and chest pain may lead to reduced energy, disturbed sleep and anxiety of the underlying cause, consequently impairing the patients' health-related quality of life. ${ }^{2}$ Long-term therapy with antisecretory agents remains the mainstay of GERD treatment. Proton pump inhibitors (PPIs) have been shown to be superior to other therapies, ${ }^{34}$ and a significant proportion of patients with GERD become dependent on PPIs for symptom control, necessitating long-term therapy. In addition, longterm PPI therapy has been postulated to increase the risk of acute and chronic kidney disease, hypomagnesaemia, Clostridium difficile infection and osteoporotic fractures. ${ }^{5}$

Other treatment options for patients with chronic GERD include laparoscopic antireflux (LAR) surgery and endoscopic antireflux interventions. ${ }^{6}$ Surgical antireflux therapies have been found to be effective in managing GERD symptoms ${ }^{7}$; however, it may lead to adverse effects such as dysphagia, bloating 
and diarrhoea, thereby affecting quality of life. ${ }^{8}$ Some patients may even continue to have GERD symptoms and remain on PPI therapy. ${ }^{9}$ Endoscopic antireflux therapies are less invasive and have been reported to reduce heartburn, regurgitation and PPI usage. Endoscopic full-thickness plication (EFTP) or endoscopic fundoplicaton involves the use of transmural sutures applied at the gastro-oesophageal junction helping restructure the anatomy of gastric cardia, thus strengthening the valvular mechanism, leading to reduced gastro-oesophageal reflux. ${ }^{810}$ To date, the largest body of evidence using EFTP is available using Esophyx device (EndoGastric Solutions, Washington, USA). In RCTS, this device has shown to be $70 \%$ effective in controlling GERD symptoms with an overall adverse event rate of $2 \%{ }^{11}$; however, the device requires advanced training, general anaesthesia and 45-100 min to complete the procedure. GERD-X (G-SURG, Germany) is a novel endoscopic plication device introduced in 2014. Compared with other EFTP devices, GERD-X appears to be simpler, less cumbersome with a shorter procedure time and safer. A previous prospective single-arm study had reported positive results on the efficacy of the GERD-X device for the treatment of patients with GERD on PPIs and refractory GERD. ${ }^{12}$ However, randomised trials and long-term follow-up data are needed to determine the efficacy and safety of the device in treating patients with GERD.

The objective of this single-centre, randomised, sham-control trial was to determine the efficacy and safety of EFTP (GERD-X) in patients with PPI-dependent GERD, compared with the sham procedure, in improving the health-related quality of life (GERDHRQL), GERD symptoms and oesophageal acid exposure.

\section{METHODS}

\section{Study design}

The study was a randomised, double-blinded sham-controlled trial conducted at the Asian Institute of Gastroenterology, India between October 2017 to April 2019. Patients with GERD symptoms, dependent on PPI therapy, were prospectively enrolled for the trial and underwent randomisation to either EFTP (GERD-X system) or a sham procedure (control). The patients and the investigator (AK) who followed the patients after intervention were blinded. However, the principle investigators (RK and DNR) who performed the EFTP procedure were aware of the treatment being provided.

\section{Patient screening and eligibility criteria}

Consecutive patients, aged 18-60 years, with classical reflux symptom (heartburn, regurgitation), dependent on PPI therapy for at least 6 months were screened for inclusion in the study. Inclusion criteria were assessed after a 7-day interruption of PPI therapy using oesophago-gastro-duodenoscopy (EGD), 24-hour $\mathrm{pH}$ impedance monitoring, and high-resolution manometry (HRM). Patients were included if they met the following criteria: gastro-oesophageal flap valve grade I-III (Hill's classification); pathological oesophageal acid exposure (percentage time with oesophageal $\mathrm{pH}<4$ in 24 hours $>4.2 \%$ ), abnormal DeMeester score $\geq 14.7$ or total reflux episodes $>73$; and lower oesophageal sphincter pressure (LESP) between 5 and $15 \mathrm{~mm} \mathrm{Hg}$. Exclusion criteria were-ASA physical status $>$ II; previous oesophageal or gastric surgery; pregnancy; large hiatal hernia $>3 \mathrm{~cm}$; Los Angeles grade C/D oesophagitis; para-oesophageal hernia; Barrett's oesophagus; and presence of oesophageal dysmotility on HRM. All the patients included in the study provided written informed consent approved by the IRB and were willing to discontinue PPI after the intervention.
During the initial screening, the duration of reflux symptoms and the dosing and duration of PPI use were recorded. After stopping PPI for 7 days, an EGD was performed to look for oesophagitis and to assess the Hill's grade of the gastrooesophageal junction and the patient's response on the GERD HRQL was assessed using GERD-HRQL validated questionnaire. ${ }^{13}$ Oesophageal HRM with 24-hour $\mathrm{pH}$ impedance monitoring was performed on day 8 of stopping PPI. The oesophageal motility pattern, LESP, $24 \mathrm{~h} \%$ time oesophageal $\mathrm{pH}<4$, number of reflux episodes, and DeMeester score were assessed at the baseline evaluation.

\section{Randomisation and intervention}

The patients included in the study were allocated to either the EFTP or the sham group in a ratio of $1: 1$ using block randomisation. The investigators narrated the procedure steps to the patients at the time of written informed consent. On the day of procedure, all the patients underwent the assigned intervention (EFTP or sham) under general anaesthesia and endotracheal intubation after overnight fasting. Assignment of the patient was sealed in an opaque envelope that was opened after anaesthetic induction and endotracheal intubation but before the introduction of gastroscope. The patients in the EFTP group were administered pre-procedure intra-venous antibiotics at the time of endoscopy. An adult gastroscope was advanced into the stomach followed by threading of a metallic guidewire through the biopsy channel of the endoscope. The gastroscope was then removed and the EFTP device was introduced over the guide-wire. Under direct endoscopic visualisation using an ultraslim endoscope, the distal end of the device was retroflexed to the anterior gastric cardia, approximately $1 \mathrm{~cm}$ below the gastro-oesophageal junction (figure 1 and online supplemental video). The arms of the device were opened; the endoscopic tissue retractor was advanced deep into the gastric cardia mucosa, which retracted the gastric wall into the open arms of the EFTP device. The arms were closed, and as per the study protocol 2 pre-tied transmural pledget sutures were deployed to ensure full-thickness plication and tight closure of gastro-oesophageal junction around the device. ${ }^{12}$ The EFTP system and the ultraslim endoscope were then straightened, removed and the gastroscope was reintroduced to evaluate the post plication gastro-oesophageal junction. No retreatments were allowed.

In the sham procedure, an identical technique was followed. The device was positioned $1 \mathrm{~cm}$ below the gastro-oesophageal junction, but the sutures were not deployed. Following the procedure, patients were placed on soft diet, and medications for pain relief were administered as needed. The patients were prescribed PPI at their preprocedure dosage, and after 7 days post procedure the patients were asked to discontinue the medications completely. As per the hospital protocol, patients were kept under observation and discharged 24 hours after the intervention.

\section{Follow-up}

After randomisation and allotted intervention, the patients were followed up and asked to visit hospital at 3, 6 and 12 months. Telephone calls were made to patients every 2-4 weeks to enquire about reflux symptoms and the investigator who made the phone call was blinded to the assignment. If the patient experienced reflux symptoms more than twice a week, PPI equivalent to $20 \mathrm{mg}$ rabeprazole or $40 \mathrm{mg}$ pantoprazole per day was prescribed over the phone and recorded. If the symptom control was inadequate at 4 weeks of starting PPI, the dose was 


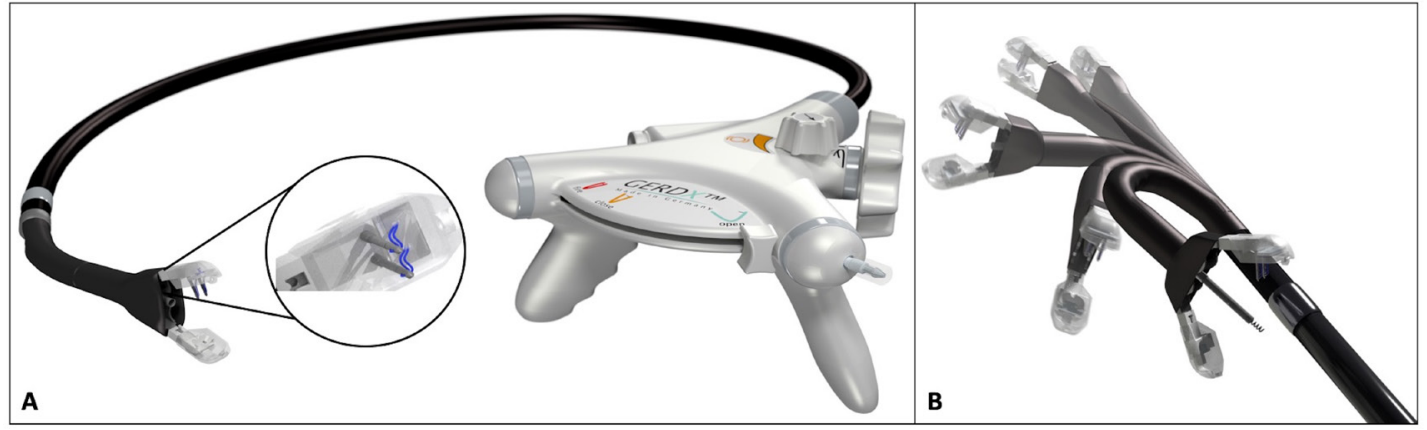

Figure 1 GERDx device $(A)$ with a control section, long shaft and two openable arms at the tip of the shaft; pretied pledget sutures are fastened to the arms (inset). (B) Retroflexion of the tip of the shaft with a rotatable tissue retractor between the arms facilitate the application of transmural sutures at the gastro-oesophageal junction.

doubled. Over the counter use of PPI was restricted but patients were allowed to use antacids on demand and noted. During each follow-up hospital visit, an EGD was performed to assess the gastro-oesophageal junction, and the GERD HRQL was assessed. In patients who resumed taking PPIs after the assigned intervention, these assessments were made after stopping PPI therapy for at least 3 days. Oesophageal HRM and 24-hour $\mathrm{pH}$ impedance monitoring were performed off PPI at 3 and 12 months after intervention.

\section{Study end points}

Primary endpoint of the study was defined as reduction of 50\% or more in GERD-HRQL total score from baseline at 3 months. Secondary end points of the study included improvement in GERD-HRQL total score, improvement in GERD symptom scores at 3, 6 and 12 months, PPI usage, and oesophageal acid exposure and reflux episodes on 24-hour $\mathrm{pH}$ impedance monitoring at 3 and 12 months. The trial was designed to have $80 \%$ power to detect a difference in GERD-HRQL at 3 months post intervention at 5\% level of significance. At an estimated rate of improvement in GERD-HRQL of 35\% in the sham group and $70 \%$ in the EFTP group, a sample size of 35 was required for each group.

\section{Definitions}

The GERD-HRQL score was calculated based on the response of the patients to the GERD-HRQL questionnaire of 16 Likerttype questions, with responses ranging from 0 (no symptoms) to 5 (worst symptoms) to each question. ${ }^{13}$ The total score was calculated by summing the individual scores to questions 1-15. The greatest possible score was 75 and the lowest was 0 . The heartburn and regurgitation scores were calculated by summing the individual scores to questions $1-6$ and $10-15$, respectively. The worst possible symptom score for each symptom was 30 and a score of $<12$ with response to each individual question not exceeding 2 indicated symptom elimination. Total reflux episodes determined by impedance were classified as acidic $(\mathrm{pH}$ $<4)$ and non-acidic $(\mathrm{pH}>4)$ based on $\mathrm{pH}$ monitoring.

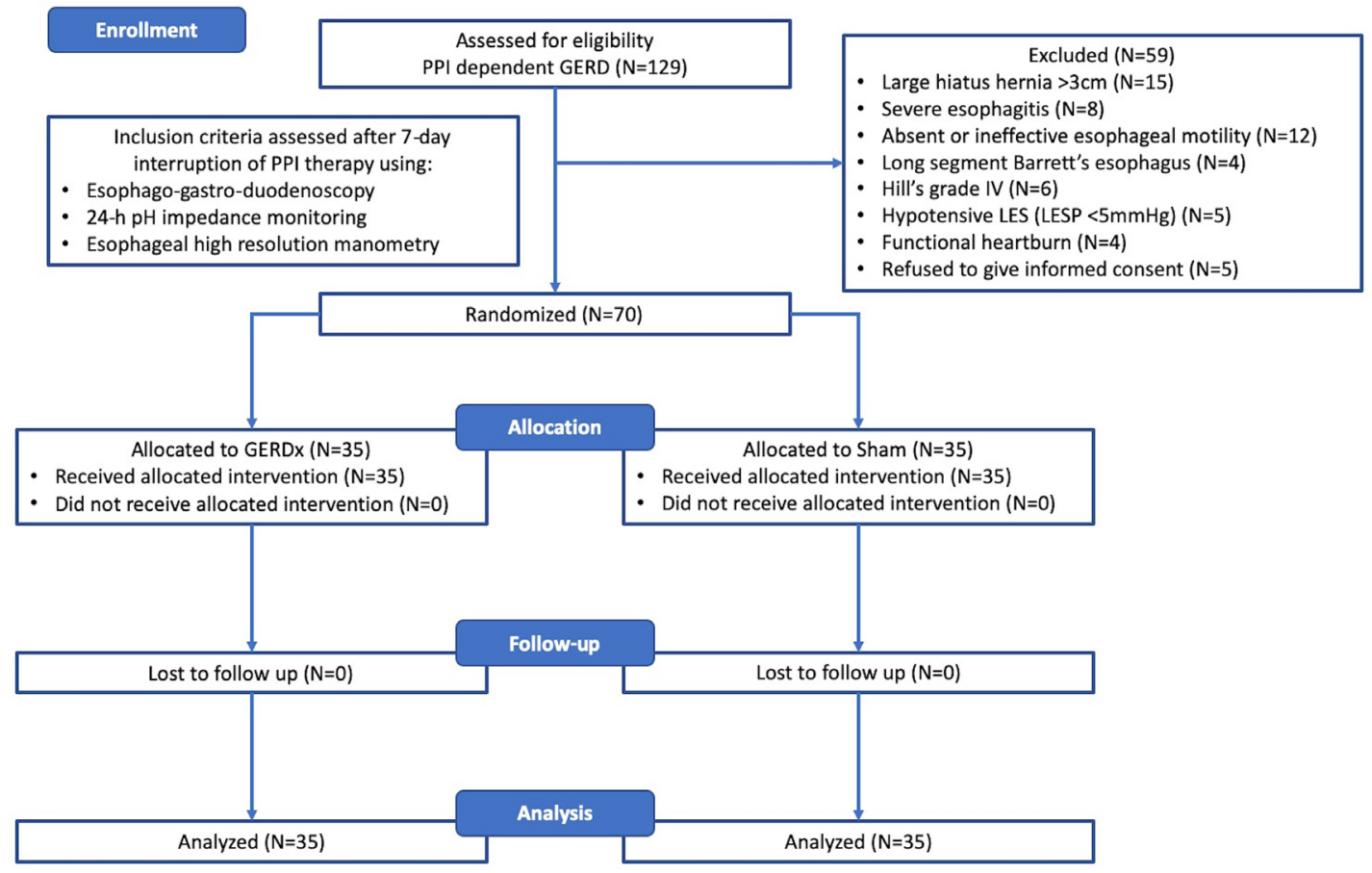

Figure 2 Consolidated Standards of Reporting Trials flow diagram showing distribution of patients in the study. PPI, proton pump inhibitor; LESP, lower oesophageal sphincter pressure; GERDx, endoscopic full-thickness fundoplication. 


\section{Adverse events}

Adverse events in the study were defined using the lexicon for endoscopic adverse events by the American society of GI endoscopy. ${ }^{14}$ Minor intraprocedural bleeding and mild postprocedural pain not requiring an intervention, premature stopping of the procedure and extended hospital stay were considered as incidents.

\section{Statistical analysis}

The continuous data were expressed as median (IQR) and compared with the Mann-Whitney $U$ test and the categorical data as frequencies and compared with the $\chi^{2}$ test. Wilcoxon signed rank test and Friedman's two-way analysis of variance by ranks were used for comparing single pairs and multiple related samples, respectively. The PPI dependency rate between the EFTP and the sham groups after intervention was compared in a Kaplan-Meier analysis using log-rank test. Cox proportional hazards model was used to estimate the HR and 95\% CIs. All tests of significance were two-tailed and a $\mathrm{p}$ value below 0.05 was considered to as statistically significant.

\section{RESULTS}

\section{Patient characteristics and procedure details}

The study design and distribution of patients are summarised in figure 2. Of 129 patients screened for eligibility, 70 met the inclusion criteria, were randomised either to the EFTP group $(n=35)$ or the sham group $(n=35)$. The median (IQR) age of the patients enrolled in the study was $36(29-42)$ years; of all the subjects, $28.6 \%$ were females and $71.4 \%$ were males. All the patients were on PPI and the median (IQR) duration of PPI use was 2.5 (1.5-4.0) years. At the time of enrolment, 5/35 (14.2\%) patients in the EFTP group and $3 / 35(8.5 \%)$ in the sham group were on double dose PPI. The baseline demographics, endoscopic, manometric and reflux parameters did not differ significantly between the EFTP and the sham groups (table 1). The mean $( \pm S D)$ duration of each procedure from the point of gastroscope insertion to the device removal was $17.4( \pm 4) \mathrm{min}$. There were no protocol deviations and all the patients in both the groups completed 12-month follow-up including per-protocol hospital visits and response to telephonic calls. Data from 24-hour $\mathrm{pH}$ impedance monitoring done off PPI at 3 months after the intervention were available for all the patients.

\section{Efficacy endpoints}

Health-related quality-of-life (GERD-HRQL) and symptom scores In the EFTP group, 65.7\% (23/35) patients achieved 50\% or more improvement in the GERD-HRQL total score at 3 months post intervention, compared with only 2.9\% (1/35) patients $(\mathrm{p}<0.001)$ in the sham group, thus meeting the primary endpoint of the study (figure 3). Results were applicable to both males (EFTP $16(64 \%)$ versus sham $0(0 \%) ; \mathrm{p}<0.001)$ and females (EFTP 7 (70\%) vs sham 1 (10\%); p 0.022) (table 2).

The median percentage improvement in the GERD-HRQL total score from baseline was significantly higher in the EFTP group compared with the sham group at 3, 6 and 12 (all $p$ values were $<0.001)$. In the EFTP group, the median percentage improvement in the GERD-HRQL total score at 6 and 12 months was significantly higher compared with that at 3 months ( $p$ values were 0.008 and $<0.001$, respectively; figure 4 ).

As compared with the sham group, the median percentage improvement in the heartburn symptom score from baseline was significantly higher in the EFTP group at 3, 6 and 12 months (all $\mathrm{p}$ values were $<0.001$ ). Similar trend was observed with

\begin{tabular}{|c|c|c|c|}
\hline & $\begin{array}{l}\text { EFTP group } \\
(n=35)\end{array}$ & $\begin{array}{l}\text { Sham group } \\
(n=35)\end{array}$ & $P$ values \\
\hline Median age (y) & $35(29-41)$ & $37(29-45)$ & 0.401 \\
\hline $\begin{array}{l}\text { Sex, } \mathbf{n}(\%) \\
\text { Female }\end{array}$ & $10(28.6 \%)$ & $10(28.6 \%)$ & 1.000 \\
\hline PPI use duration (y) & $2.0(1.0-5.0)$ & $2.5(1.5-3.5)$ & 0.855 \\
\hline \multicolumn{4}{|l|}{ Hill's grade of GE flap, $n(\%)$} \\
\hline I & $16(45.7)$ & $18(51.4)$ & 0.746 \\
\hline ॥ & $6(17.1)$ & $7(20)$ & \\
\hline III & $13(37.1)$ & $10(28.6)$ & \\
\hline \multicolumn{4}{|l|}{ Oesophagitis LA grade, $n(\%)$} \\
\hline Normal & $24(68.6)$ & $25(71.4)$ & 0.543 \\
\hline A & $11(31.4)$ & $9(25.7)$ & \\
\hline B & $0(0)$ & $1(2.9)$ & \\
\hline $\begin{array}{l}\text { Baseline median } \% \text { time } \\
\text { oesophageal } \mathrm{pH}<4\end{array}$ & $4.4(2.0-8.7)$ & $2.7(1.3-5.9)$ & 0.127 \\
\hline DeMeester score & $15.1(7.6-28.0)$ & $12.2(6.8-21.4)$ & 0.447 \\
\hline Total reflux episodes & $90(65-115)$ & $92(65-130)$ & 0.733 \\
\hline Acid reflux episodes & $51(33-73)$ & $40(27-61)$ & 0.106 \\
\hline Non acid reflux episodes & $34(18-71)$ & $49(25-77)$ & 0.264 \\
\hline GERD HRQL total score & $40(23-51)$ & $43(30-50)$ & 0.492 \\
\hline Heart Burn symptom score & $19(12-26)$ & $18(8-26)$ & 0.597 \\
\hline Regurgitation symptom score & $21(10-26)$ & $24(19-28)$ & 0.076 \\
\hline
\end{tabular}

All continuous data are expressed as median (IQR).

EFTP, endoscopic full-thickness plication; GE, gastro-oesophageal; GERD-HRQL, GERD health-related quality of life; LA, Los Angeles classification of erosive oesophagitis; PPI, proton pump inhibitors.

percentage improvement of regurgitation symptom score from baseline in the EPTF group compared with the sham group at 3, 6 and 12 months (all p values were $<0.001$; figure 5). Within the EFTP group, the median percentage improvement in the heartburn and regurgitation symptom scores at 12 months was significantly higher than that at 3 months ( $p$ values were 0.005 and 0.001 , respectively). At 12 months post intervention, a significantly higher proportion of patients in the EFTP group, compared with the sham group, had elimination of heartburn $(97.1 \%(34 / 35)$ vs $48.6 \%(17 / 35) ; \mathrm{p}<0.001)$ and regurgitation (94.3\% (33/35) vs $22.9 \%(8 / 35) ; \mathrm{p}<0.001)$.

Endoscopic evaluation at 3, 6 and 12 months showed Hill's grade 1 in 100\%, 91.5\% and 77.8\%, respectively, in EFTP group, in sham arm endoscopic Hill's grade was the same as baseline. Mucosal wrap and suture were intact in all patients at 12 months. There was no symptomatic dysphagia nor endoscopic evidence of luminal narrowing at the GE junction (online supplemental table 1). At 12 months, endoscopy showed no oesophagitis was seen in all EFTP $(n=18)$ patients; in sham arm, 29.4\% (5/17) patients had grade A oesophagitis.

\section{PPI use}

The PPI dependence at 12 months after intervention was significantly higher in the sham group compared with the EFTP group. A total of $22(62.8 \%)$ patients in the EFTP group and 4 $(11.4 \%)$ patients in the sham group were off PPI at the end of the study $(\mathrm{p}<0.001)$. The Kaplan-Meier survival analysis showed a significantly lower cumulative probability of PPI dependence in the EFTP group compared with the sham group over time after intervention (HR, 0.25 ; 95\% CI 0.12 to 0.49 ; $\mathrm{p}<0.001$; figure 6). In the EFTP group, of five patients on double dose PPI at baseline, two resumed taking double dose PPI while the other three patients were off PPI at 12 months after the intervention. 

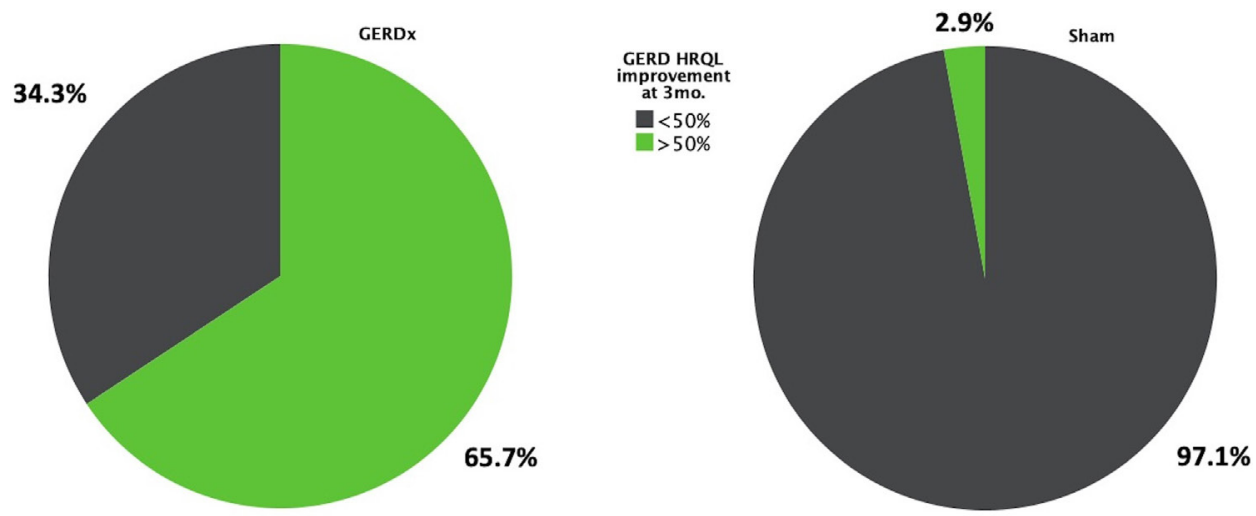

Figure 3 Primary end point. The endoscopic full-thickness plication (EFTP; GERDx) group achieved $\geq 50 \%$ improvement in GERD-HRQL total score more frequently than the sham group at 3 months post intervention. GERD-HRQL, GERD health-related quality of life; GERDx or EFTP, endoscopic fullthickness fundoplication.

Rest of the patients (11/35) in the EFTP group who resumed taking PPI after the intervention were on single-dose PPI. In the sham group, all three patients on double-dose PPI at the time of enrolment, resumed taking the same dose of PPI at 12 months after the procedure. Additionally, in two patients who were on single-dose PPI before the sham procedure, PPI dose had to be doubled after the procedure for symptom control.

\section{4-hour ambulatory pH impedance study}

The 24-hour $\mathrm{pH}$ impedance study done at $3(\mathrm{n}=70$; EFTP $=35$ and sham =35) and $12(\mathrm{n}=27 ; \mathrm{EFTP}=27$ and sham =9) months after the intervention showed a reduction in the oesophageal acid exposure (median percentage time with $\mathrm{pH}<4$ in 24 hours) from baseline in the EFTP group; however, the difference did not reach statistical significance. In addition, the median percentage time with oesophageal $\mathrm{pH}<4$ and the DeMeester score were comparable between the EFTP and the sham groups at 3 and 12 months (table 3 ).

A significant reduction in the median number of total reflux episodes recorded in 24 hours was observed after the intervention in the EFTP group at 3 months from baseline (figure 7), with trend towards improvement at 12 months. A trend towards fewer total reflux episodes in 24 hours was noted in the EFTP group compared with that in the sham group at 3 months ( $p$ 0.072 ) and 12 months ( $p$ 0.051). Moreover, the median number of non-acid reflux episodes in 24 hours was significantly lower in the EFTP group compared with the sham group at 3 months (p 0.048) and 12 months (p 0.005) after intervention. There was significant decrease in non-acid reflux episodes in EFTP arm at 12 months compared with baseline ( $p$ 0.038). There was no significant difference in the median number of acid reflux episodes in 24 hours between the EFTP and the sham groups.

\section{Adverse events}

There were no major procedure-related adverse events. Mild and moderate adverse events in the EFTP were noted in one patient each. The adverse events in the EFTP group included left-sided chest pain requiring analgesics and left-sided pleural effusion along with fever in one patient each. CT with oral contrast did not show any contrast leak in both the patients. The patient with chest pain improved with analgesics alone whereas the case with pleural effusion was managed with intravenous antibiotics and required an extended stay for 5 days. There were no adverse events in the sham group.

Other incidents (not considered as adverse events) included transient intraoperative bleeding at the site of suture application in 5/35 (14.2\%) and left shoulder pain in 8/35 (22.8\%) (table 4).

At 3-month follow-up, no delayed adverse events related to the procedure were observed in both groups.

\section{DISCUSSION}

In this randomised, sham-controlled, single-blinded clinical trial, we evaluated the efficacy and safety of endoscopic full thickness fundoplication using a novel device for patients with GERD. We included PPI-dependent patients with classic reflux symptoms

Table 2 Comparison of symptom scores between endoscopic full-thickness plication (EFTP) and sham groups at 3, 6 and 12 months after intervention

\begin{tabular}{|c|c|c|c|c|}
\hline Parameter & & $\operatorname{EFTP}(n=35)$ & Sham $(n=35)$ & $P$ value \\
\hline \multirow[t]{3}{*}{ GERD-HRQL, Median percentage improvement } & 3 Months & $69.3(38.0-87.2)$ & $6.6(2.1-13.9)$ & 0.001 \\
\hline & 6 Months & $81.4(60.9-100)$ & $8.0(2.2-21.6)$ & 0.001 \\
\hline & 12 Months & $92.3(84.4-100)$ & $9.1(4.8-36.0$ & 0.001 \\
\hline \multirow[t]{3}{*}{ Heart Burn symptom Score, Median percentage improvement } & 3 Months & $55.6(37.9-100)$ & $7.4(0-23.1)$ & 0.001 \\
\hline & 6 Months & $75.0(56.5-100)$ & $13(0-37.5)$ & 0.001 \\
\hline & 12 Months & $89.7(66.7-100)$ & $15.4(0-44.4)$ & 0.001 \\
\hline \multirow[t]{3}{*}{ Regurgitation symptom score, median percentage improvement } & 3 Months & $60(47.6-92.3)$ & $7.7(0-13.3)$ & 0.001 \\
\hline & 6 Months & $96.2(60-100)$ & $6.9(0-17.9)$ & 0.001 \\
\hline & 12 Months & $100(90-100)$ & $3.4(-3.4$ to 27.3$)$ & 0.001 \\
\hline
\end{tabular}

All continuous data are expressed as median (IQR).

EFTP, endoscopic full-thickness plication; GERD-HRQL, GERD health-related quality of life 


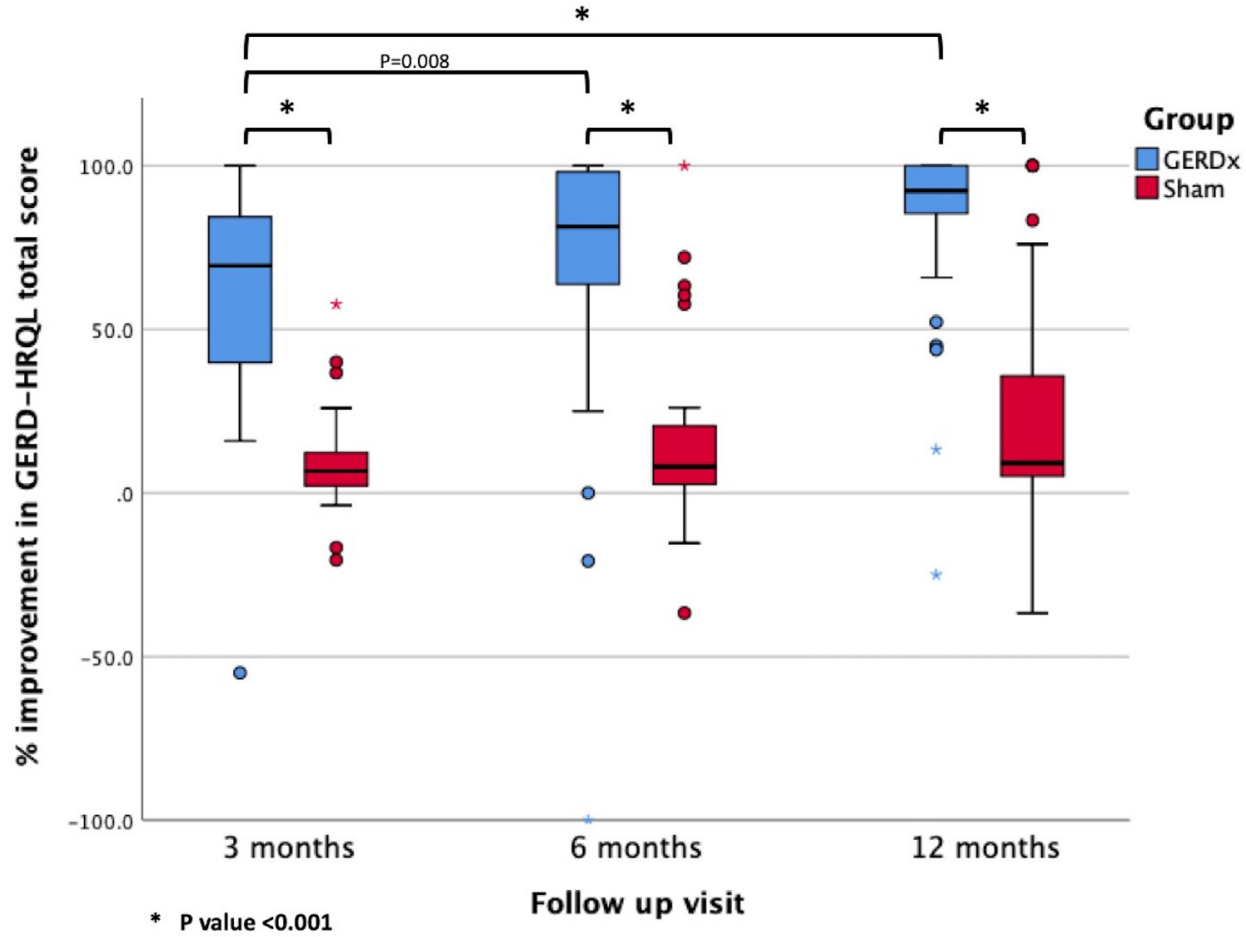

Figure $4 \%$ improvement in GERD-HRQL total score. The EFTP (GERDx) group had significant improvement in the GERD-HRQL total score at 3, 6 and 12 months post intervention compared to the sham group. Error bars indicate $95 \% \mathrm{Cl}$. Red and blue dots and asterisks represent outliers. GERDHRQL, GERD health-related quality of life; GERDx or EFTP, endoscopic full-thickness fundoplication.

well controlled on therapy. All the patients had moderate to severe degree of reflux symptoms as assessed by the GERDHRQL questionnaire after stopping PPI therapy. We objectively confirmed the diagnosis of GERD based on abnormal acid reflux or increased number of reflux episodes on 24-hour $\mathrm{pH}$ impedance study and excluded patients with functional heartburn. We showed that the device used for endoscopic fundoplication in our study was safe with no major complications and the operating time was short (mean time $17.4 \mathrm{~min}$ ). There was significant improvement in the GERD-HRQL score at 3 and 12 months. However, 24-hour $\mathrm{pH}$ impedance studies showed partial improvement at 3 months and not at 12 month. At 12 months, in EFTP group, there was significant reduction in non-acid reflux compared with baseline and sham arm. A significant proportion of patients (63\%) discontinued taking PPI in the EFTP group compared with those in the sham group (11\%) at the end of the study $(\mathrm{p}<0.001)$.

The primary outcome $(50 \%$ or more improvement in GERDHRQL total score) was more frequently observed in the EFTP group $(65.7 \%)$ compared with the sham group (3\%) at 3 months after intervention $(p<0.001)$. Our short-term results were slightly superior to those from a study that evaluated the efficacy of a similar but older plicator device (NDO Surgical). ${ }^{15}$ In the NDO plicator group, $56 \%$ of patients achieved $>50 \%$ improvement in GERD-HRQL score at 3 months compared with $18.5 \%$ in the sham group. Randomised controlled trials
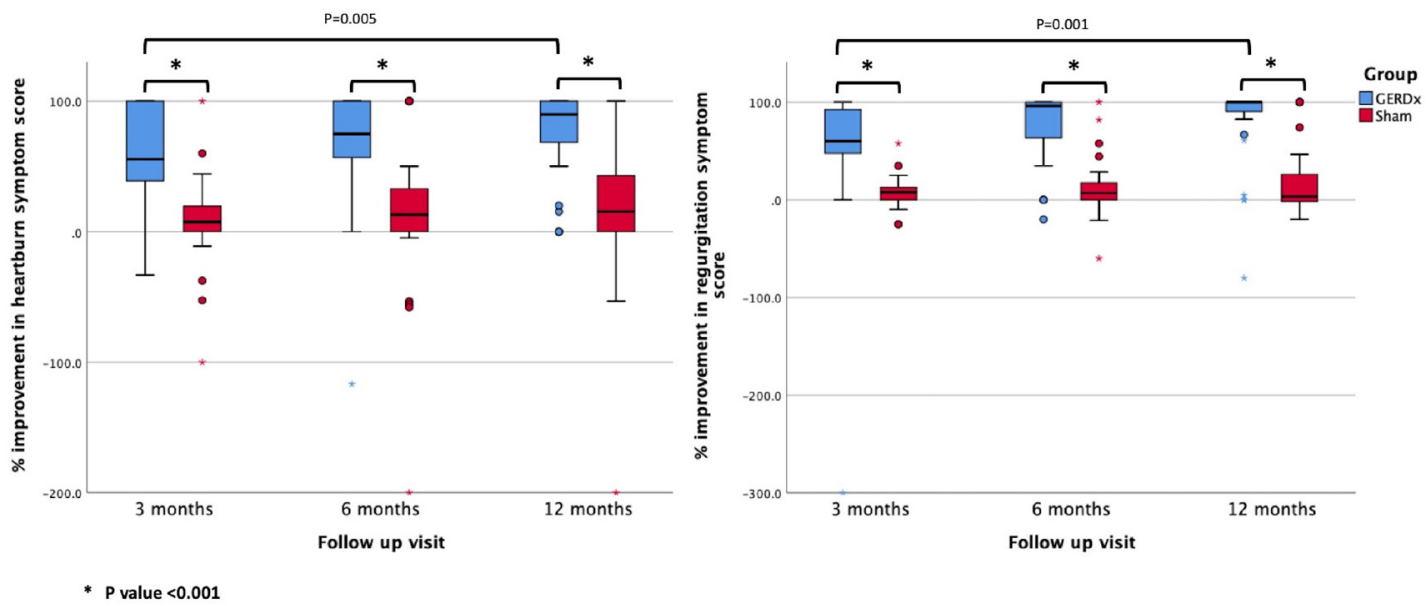

Figure $5 \%$ improvement in reflux symptom scores. The EFTP (GERDx) group had significant improvement in heartburn and regurgitation scores at 3, 6 and 12 months post intervention compared to the sham group. Error bars indicate $95 \% \mathrm{Cl}$. Red and blue dots and asterisks represent outliers. GERD-HRQL, GERD health-related quality of life; GERDx or EFTP, endoscopic full-thickness fundoplication. 


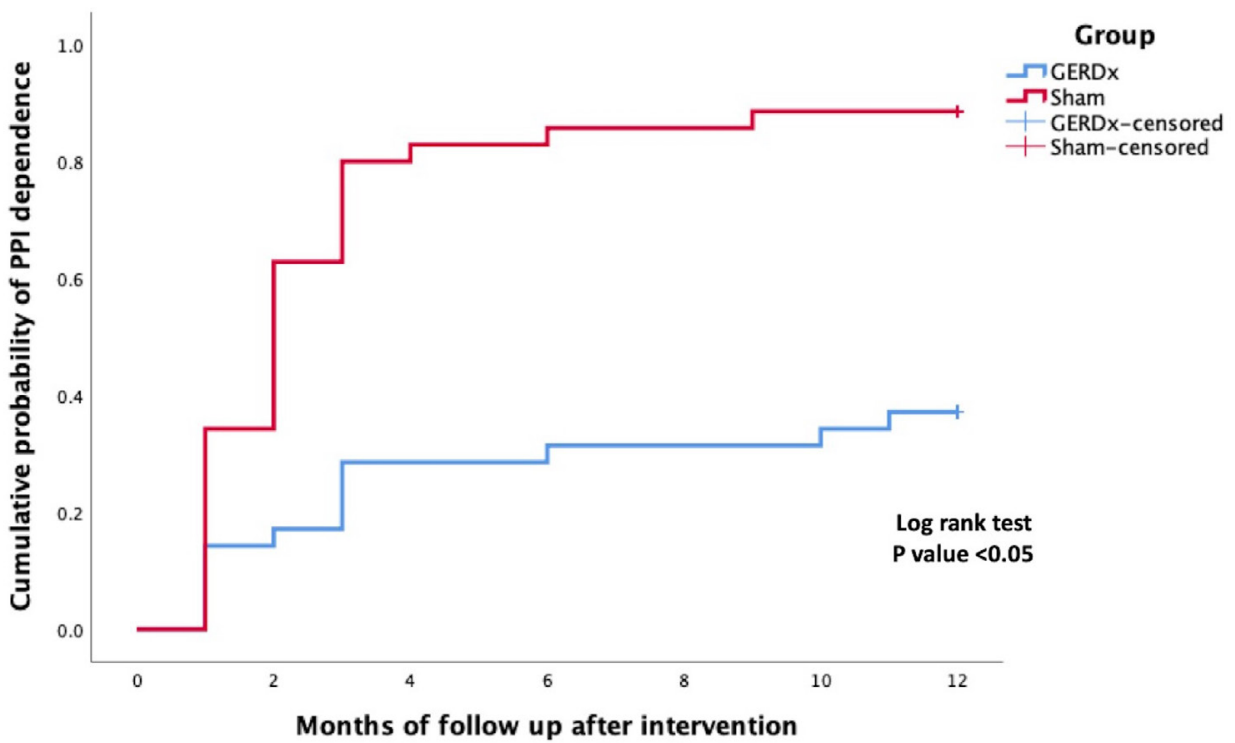

Number at risk

$\begin{array}{lccccccc}\text { GERDX } & 35 & 30 & 25 & 25 & 24 & 24 & 22 \\ \text { Sham } & 35 & 23 & 7 & 6 & 5 & 4 & 4\end{array}$

Figure 6 Proton pump inhibitors (PPIs) dependency. The Kaplan-Meier survival analysis showed a significantly lower cumulative probability of PPI dependence in the EFTP (GERDx) group compared to the sham group over time after intervention. GERDx or EFTP, endoscopic full-thickness fundoplication.

evaluating the efficacy of transoral incisionless fundoplication (TIF) have reported similar short-term outcomes. ${ }^{16}$ Using a similar outcome measure, Witteman et al reported an improvement in the GERD-HRQL score in $55 \%$ of patients at 6 months after TIF. ${ }^{17}$ Another prospective, non-comparative trial evaluating the clinical feasibility of the GERD-X device demonstrated significant improvement in mean reflux-specific symptom scores at 3 months ${ }^{12}$; however, the study lacked a control arm and the efficacy beyond 3 months was not reported.
The GERD-HRQL total score in the EFTP group improved progressively after intervention and the degree of improvement at 6 and 12 months was superior to that at 3 months. Similar trend of improvement was observed with heartburn and regurgitation symptom scores. On endoscopy, there was significant and sustained improvement in Hill's grade with intact mucosal wrap and sutures up to 12 months in EFTP group. There was no objective evidence of luminal narrowing at GE junction or erosive oesophagitis, also none of patients complained of dysphagia.

Table 3 Comparison of 24-hour pH impedance study results between endoscopic full-thickness plication (EFTP) and sham groups at 3 and 12 months after intervention

\begin{tabular}{|c|c|c|c|c|c|c|}
\hline Parameter & & EFTP & $\begin{array}{l}\text { P value } \\
\text { Baseline versus } 3 \text { and } \\
12 \text { months }\end{array}$ & Sham & $\begin{array}{l}\text { P value } \\
\text { Baseline versus } \\
3 \text { and } 12 \text { months }\end{array}$ & $\begin{array}{l}\text { P value } \\
\text { EFTP versus } \\
\text { sham }\end{array}$ \\
\hline \multirow[t]{2}{*}{ Median $\%$ time oesophageal $\mathrm{pH}<4$} & Baseline & $4.4(2.0-8.7)$ & & $2.7(1.3-5.9)$ & & 0.127 \\
\hline & 12 months & $3.4(0.6-5.5)$ & 0.276 & $5.4(2.0-9.0)$ & 0.441 & 0.111 \\
\hline DeMeester Score & Baseline & $15.1(7.6-28.0)$ & & $12.2(6.8-21.4)$ & & 0.447 \\
\hline \multirow[t]{3}{*}{ Total reflux episodes } & Baseline & $90(65-115)$ & & $92(65-130)$ & & 0.733 \\
\hline & 3 months & $66(46-92)$ & 0.005 & $85(61-122)$ & 0.055 & 0.072 \\
\hline & 12 months & $54.5(33-100)$ & 0.122 & $120(69.5-129)$ & 0.859 & 0.051 \\
\hline \multirow[t]{2}{*}{ Acid reflux episodes } & Baseline & $51(33-73)$ & & $40(27-61)$ & & 0.106 \\
\hline & 3 months & $34(16-63)$ & 0.063 & $40(30-62)$ & 0.933 & 0.414 \\
\hline
\end{tabular}

All continuous data are expressed as median (IQR). 

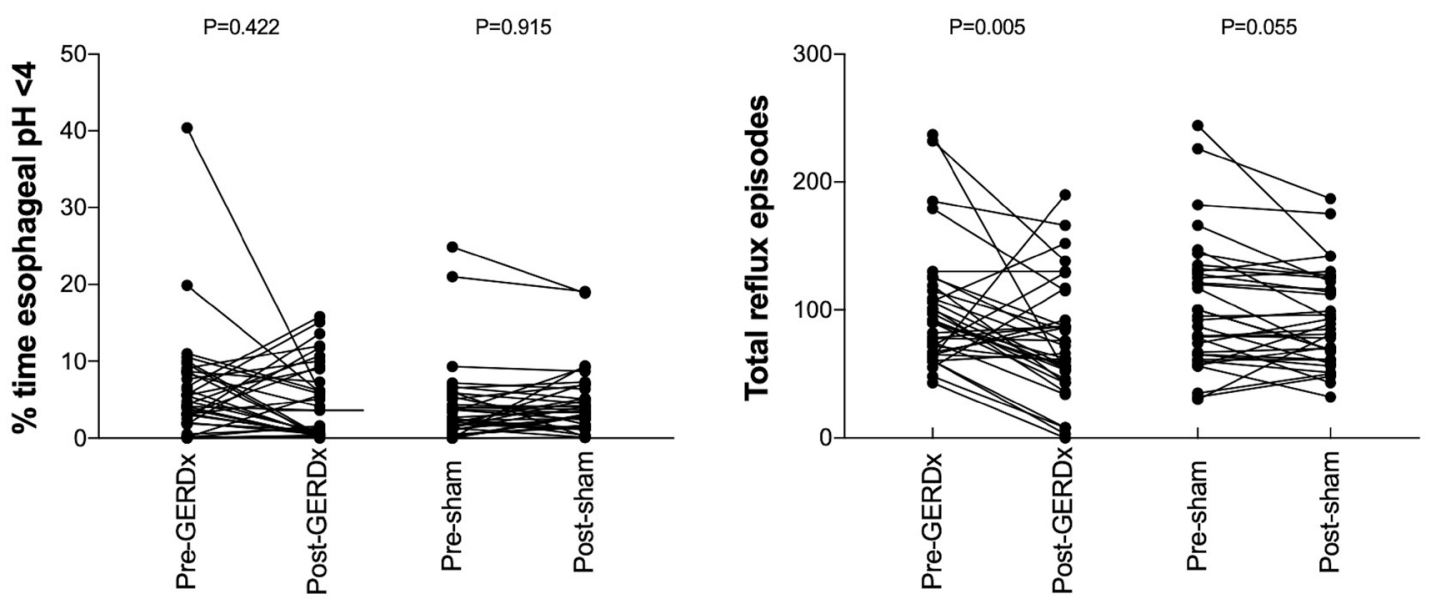

Figure 7 24-hour reflux parameters at 3 months post intervention. Before and after plots showing significant reduction in total reflux episodes, but not acid exposure time in the EFTP (GERDx) group compared to the sham group. GERDx or EFTP, endoscopic full-thickness fundoplication.

The majority of the trials that evaluated the efficacy of LAR surgery indicate that the proportion of patients experiencing reflux symptoms decreased drastically over the first year after the surgery $\left(80 \%-90 \%\right.$ at baseline to $2 \%-4 \%$ at the end of 1 year). ${ }^{18}$ Long-term follow-up studies of LAR surgery have shown that the reduction in the symptom severity was marked at 6 months and 1 year but a sizeable proportion of patients experienced a relapse after 5 years $(14 \%-18 \%)$ and 10 years $(30 \%-35 \%){ }^{7}$ Studies that evaluated the long-term efficacy of TIF have shown similar results. ${ }^{19} 20$ Stefanidis et al reported significant improvement in regurgitation and chest pain scores at 12 months compared with baseline and at 6 months after TIF using Esophyx device. ${ }^{21}$ In a few open-label randomised controlled trials comparing TIF with PPI, the mean GERD-HRQL score dropped significantly at the end of 1 year (27-32 at baseline to 7-10 at 12 months) in the TIF arm. ${ }^{1722}$ Data from the above studies suggest that the effects of surgical or endoscopic fundoplication are progressive over a year or two after the intervention and decline thereafter. Since the EFTP device used in our study was a novel one and short-term data on its efficacy were lacking as well, we looked at the trend of events at regular time intervals starting from 3 months post intervention. Moreover, the 12 -month outcome in our study was far superior to those of the previous studies evaluating an identical plicator device. ${ }^{23-26}$

The PPI dependence rate was significantly higher in the sham group compared with the EFTP group. Of 13 patients who resumed taking PPI in the EFTP group, 11 (85\%) did so during the first 3 months after the intervention. Studies evaluating TIF using Esophyx device have shown similar results. In a prospective non-comparative trial, $80 \%$ of the patients who were dependent on PPI at the end of 12 months, restarted taking PPI within 6 months after TIF. ${ }^{19}$ In the majority of the studies that evaluated the efficacy of an older plicator device in PPI dependent patients, $60 \%-70 \%$ of patients were off PPI at 6 and 12 months after intervention. ${ }^{23}{ }^{27}$ However, most of these studies were nonrandomised and did not have a control arm.

24-hour $\mathrm{pH}$ impedance study done at 3 months after the intervention showed an improvement in oesophageal acid exposure in the EFTP group from baseline; however, the difference was not statistically significant. On the other hand, the number of total and non-acid reflux episodes decreased in the EFTP group compared with the sham group at 3 and 12 months. This suggests that clinical response to EFTP may be due to reduction in volume reflux as evident by a significant reduction in the total number of reflux episodes rather than normalisation of acid exposure time. The results of our study are in concordance to previous studies where a reduction in oesophageal acid exposure has been found, but the normalisation of distal oesophageal acid exposure was infrequent.$^{11} 17$ Improvement in GERD-HRQL may be a more relevant and patient centric goal as compared with normalisation of oesophageal acid exposure which is infrequent with the currently available devices. The current study has showed that there is significant improvement in symptoms and less improvement in objective parameters as per $24 \mathrm{pH}$ impedance study, mostly in patients with non-erosive oesophagitis (NERD).

As compared with other endoscopic antireflux procedures, a relatively short operating time could be a valuable feature of this novel EFTP device. Shorter operating time is one of the surrogate markers of the technical ease of the procedure. Except for few adverse events related to suture characteristics, EFTP is a safe procedure and does not require prolonged hospitalisation. In addition, we presume that the procedure has a low learning curve and performing initial 10-15 procedures under supervision is sufficient to gain adequate experience.

As per our knowledge, our study is the first randomised, sham controlled trial evaluating the efficacy of this novel EFTP device in PPI dependent patients with GERD. Patients were blinded

\begin{tabular}{llllllll}
\hline Table 4 & \multicolumn{6}{l}{ Summary of procedure related events reported during the study } & \\
\hline Event no. & Group & Nature & Category & Description & Frequency & Length of hospital stay (d) & Severity \\
\hline 1 & EFTP & AE & Pain & Left sided chest pain & $1 / 35(2.8 \%)$ & 3 & Mild \\
2 & EFTP & Incident & & Left shoulder pain & $8 / 35(22.8 \%)$ & 1 & - \\
3 & EFTP & AE & Pulmonary & Fever, moderate left sided pleural effusion & $1 / 35(2.8 \%)$ & 5 & Moderate \\
4 & EFTP & Incident & Bleeding & Intraoperative bleeding at the site of suture application & $5 / 35(14.2 \%)$ & 1 & - \\
\hline
\end{tabular}

$A E$, adverse event; EFTP, endoscopic full-thickness plication. 
for the nature of intervention and were meticulously followed up for 12 months. However, few limitations are noteworthy. First, our study was conducted at a single centre and included a small sample size. Second, initial screening and enrolment of PPI-dependent patients were based on historical details and PPI dependency was not confirmed objectively. Third, the reflux was not assessed objectively at the end of 12-month follow-up in all patients; we could perform $24 \mathrm{~h} \mathrm{pH}$ impedance study in 27 (38.57\%, 18 in EFTP and 9 in sham arm) patients.

In summary, in our randomised, single-blinded, shamcontrolled trial, endoscopic full thickness fundoplication procedure using a novel device was found effective at reducing the GERD symptoms and improving the quality of life; thought without objective 24-hour $\mathrm{pH}$ impedance study. This endoluminal procedure is a promising alternative option to surgery in appropriately selected group of patients, who may not want to continue PPI long term. Preferred cohort of patients who would benefit from EFTP includes those with PPI dependence, abnormal acid or non-acid reflux and small hiatus hernia. The procedure is short and with very few side effects. Large, prospective trials with long-term follow-up are required to conclude the benefits of this procedure after 1 year.

\section{Author affiliations}

'Medical Gastroenterology, Asian Institute of Gastroenterology, Hyderabad, Telangana, India

${ }^{2}$ Anaesthesia, Asian Institute of Gastroenterology, Hyderabad, Telangana, India ${ }^{3}$ Medical Gastroenterology, Asian Institute of Gastroenterology, Hyderabad, India ${ }^{4}$ Gastroenterology, Asian Institute of Gastroenetrology, Hyderabad, Andhra Pradesh, India

${ }^{5}$ Asian Institute of Gatroenterology, Hyderabad, India

${ }^{6}$ Asian Institute of Gastroenterology, Hyderabad, Telangana, India

${ }^{7}$ Surgical Gastroenterology, Asian Institute of Gastroenterology, Hyderabad, India

${ }^{8}$ University of Kansas, Kansas City, Kansas, USA

${ }^{9}$ Asian Healthcare Foundation, Asian Institute of Gastroenterology, Hyderabad, Andhra Pradesh, India

Twitter Rakesh Kalapala @drkalpala

Contributors Concept: RK, AK, DNR. Design: RK, AK, MR, PS. Supervision: RG, SD, SL, GVR. Resources: RK, AK, RG, SD. Data collection and/or processing: AK, RG, RK. Analysis and/or interpretation: AK, RK, PS, NJ. Literature search, writing manuscript: AK, RK. Critical review: RG, GVR, DNR, PS. Final approval: all authors.

Funding The authors have not declared a specific grant for this research from any funding agency in the public, commercial or not-for-profit sectors.

Competing interests None declared.

Patient consent for publication Not required.

Ethics approval Asian Institute of Gastroenterology, Institutional Ethics Committee AIG/IEC30/09.2017-01.

Provenance and peer review Not commissioned; externally peer reviewed.

Data availability statement Data are available upon reasonable request. We will share the data on request.

Supplemental material This content has been supplied by the author(s). It has not been vetted by BMJ Publishing Group Limited (BMJ) and may not have been peer-reviewed. Any opinions or recommendations discussed are solely those of the author(s) and are not endorsed by BMJ. BMJ disclaims all liability and responsibility arising from any reliance placed on the content. Where the content includes any translated material, BMJ does not warrant the accuracy and reliability of the translations (including but not limited to local regulations, clinical guidelines, terminology, drug names and drug dosages), and is not responsible for any error and/or omissions arising from translation and adaptation or otherwise.

Open access This is an open access article distributed in accordance with the Creative Commons Attribution Non Commercial (CC BY-NC 4.0) license, which permits others to distribute, remix, adapt, build upon this work non-commercially, and license their derivative works on different terms, provided the original work is properly cited, appropriate credit is given, any changes made indicated, and the use is non-commercial. See: http://creativecommons.org/licenses/by-nc/4.0/.

\section{ORCID iDs}

Rakesh Kalapala http://orcid.org/0000-0003-3203-7708
Nitin Jagtap http://orcid.org/0000-0002-3469-0164

\section{REFERENCES}

1 El-Serag HB, Sweet S, Winchester CC, et al. Update on the epidemiology of gastrooesophageal reflux disease: a systematic review. Gut 2014;63:871-80.

2 Wiklund I. Review of the quality of life and burden of illness in gastroesophageal reflux disease. Dig Dis 2004;22:108-14.

3 Lowe RC, Wolfe MM. The pharmacological management of gastroesophageal reflux disease. Minerva Gastroenterol Dietol 2004;50:227-37.

4 Spechler SJ, Lee E, Ahnen D, et al. Long-Term outcome of medical and surgical therapies for gastroesophageal reflux disease: follow-up of a randomized controlled trial. JAMA 2001;285:2331-8.

5 Kinoshita Y, Ishimura N, Ishihara S. Advantages and disadvantages of long-term proton pump inhibitor use. J Neurogastroenterol Motil 2018;24:182-96.

6 Nabi Z, Reddy DN. Update on endoscopic approaches for the management of gastroesophageal reflux disease. Gastroenterol Hepatol 2019;15:369-76.

7 Lundell L, Bell M, Ruth M. Systematic review: laparoscopic fundoplication for gastroesophageal reflux disease in partial responders to proton pump inhibitors. World I Gastroenterol 2014:20:804-13.

8 Antoniou SA, Koch 00, Kaindlstorfer A, et al. Endoscopic full-thickness plication versus laparoscopic fundoplication: a prospective study on quality of life and symptom control. Surg Endosc 2012;26:1063-8.

9 Bammer T, Hinder RA, Klaus A, et al. Five- to eight-year outcome of the first laparoscopic Nissen fundoplications. J Gastrointest Surg 2001;5:42-8.

10 Chen YK, Raijman I, Ben-Menachem T, et al. Long-Term outcomes of endoluminal gastroplication: a U.S. multicenter trial. Gastrointest Endosc 2005;61:659-67.

11 McCarty TR, Itidiare M, Njei B, et al. Efficacy of transoral incisionless fundoplication for refractory gastroesophageal reflux disease: a systematic review and meta-analysis. Endoscopy 2018;50:708-25.

12 Weitzendorfer M, Spaun GO, Antoniou SA, et al. Clinical feasibility of a new fullthickness endoscopic plication device (GERDX ${ }^{T M}$ ) for patients with GERD: results of a prospective trial. Surg Endosc 2018;32:2541-9.

13 Velanovich $\mathrm{V}$. The development of the GERD-HRQL symptom severity instrument. Dis Esophagus 2007;20:130-4.

14 Cotton PB, Eisen GM, Aabakken L, et al. A lexicon for endoscopic adverse events: report of an ASGE workshop. Gastrointest Endosc 2010;71:446-54.

15 Rothstein R, Filipi C, Caca K, et al. Endoscopic full-thickness plication for the treatment of gastroesophageal reflux disease: a randomized, sham-controlled trial. Gastroenterology 2006;131:704-12.

16 Håkansson B, Montgomery M, Cadiere GB, et al. Randomised clinical trial: transoral incisionless fundoplication vs. sham intervention to control chronic GERD. Aliment Pharmacol Ther 2015;42:1261-70.

17 Witteman BPL, Conchillo JM, Rinsma NF, et al. Randomized controlled trial of transoral incisionless fundoplication vs. proton pump inhibitors for treatment of gastroesophageal reflux disease. Am J Gastroenterol 2015;110:531-42.

18 Galmiche J-P, Hatlebakk J, Attwood S, et al. Laparoscopic antireflux surgery vs esomeprazole treatment for chronic GERD: the Lotus randomized clinical trial. JAMA 2011;305:1969-77

19 Wilson EB, Barnes WE, Mavrelis PG, et al. The effects of transoral incisionless fundoplication on chronic GERD patients: 12-month prospective multicenter experience. Surg Laparosc Endosc Percutan Tech 2014;24:36-46.

20 Testoni PA, Testoni S, Mazzoleni G, et al. Long-term efficacy of transoral incisionless fundoplication with Esophyx (Tif 2.0) and factors affecting outcomes in GERD patients followed for up to 6 years: a prospective single-center study. Surg Endosc 2015:29:2770-80.

21 Stefanidis G, Viazis N, Kotsikoros N, et al. Long-Term benefit of transoral incisionless fundoplication using the EsophyX device for the management of gastroesophageal reflux disease responsive to medical therapy. Dis Esophagus 2017;30:1-8.

22 Trad KS, Fox MA, Simoni G, et al. Transoral fundoplication offers durable symptom control for chronic GERD: 3-year report from the tempo randomized trial with a crossover arm. Surg Endosc 2017;31:2498-508.

23 von Renteln D, Schiefke I, Fuchs KH, et al. Endoscopic full-thickness plication for the treatment of gastroesophageal reflux disease using multiple Plicator implants: 12-month multicenter study results. Surg Endosc 2009;23:1866-75.

24 Chuttani R, Sud R, Sachdev G, et al. A novel endoscopic full-thickness plicator for the treatment of GERD: a pilot study. Gastrointest Endosc 2003;58:770-6.

25 Pleskow D, Rothstein R, Lo S, et al. Endoscopic full-thickness plication for the treatment of GERD: a multicenter trial. Gastrointest Endosc 2004;59:163-71.

26 Pleskow D, Rothstein R, Kozarek R, et al. Endoscopic full-thickness plication for the treatment of GERD: five-year long-term multicenter results. Surg Endosc 2008;22:326-32

27 Koch 00, Kaindlstorfer A, Antoniou SA, et al. Subjective and objective data on esophageal manometry and impedance $\mathrm{pH}$ monitoring 1 year after endoscopic fullthickness plication for the treatment of GERD by using multiple plication implants. Gastrointest Endosc 2013;77:7-14. 